PAPER • OPEN ACCESS

\title{
Powder diffraction computed tomography: a combined synchrotron and
} neutron study

To cite this article: Vladislav Kochetov et al 2021 J. Phys.: Condens. Matter 33105901

View the article online for updates and enhancements.

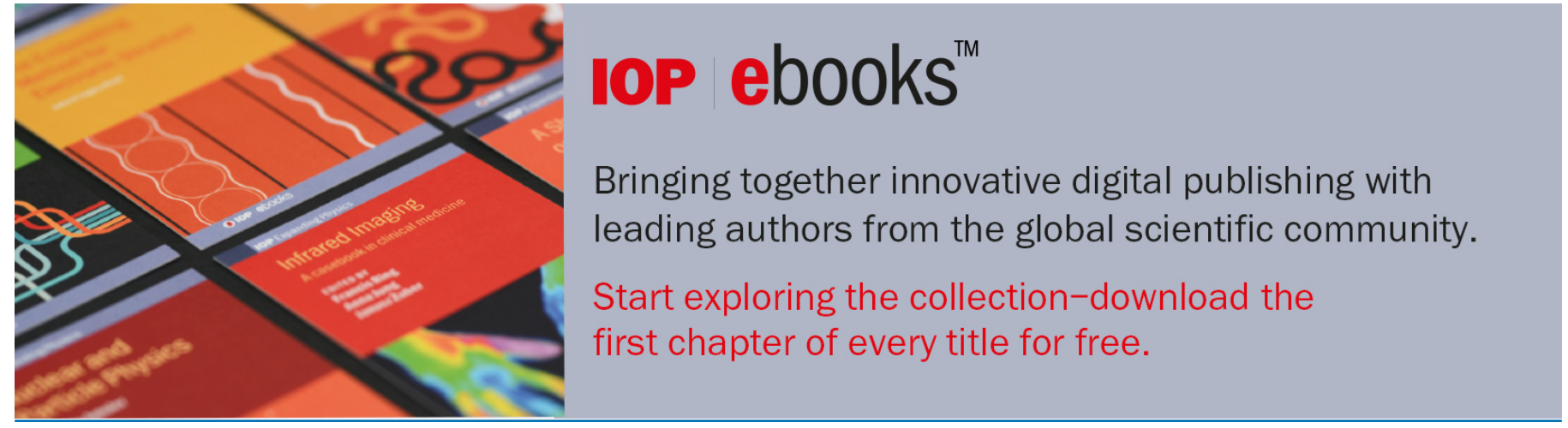

This content was downloaded from IP address 129.13 .72 .197 on 05/02/2021 at 13:51 


\title{
Powder diffraction computed tomography: a combined synchrotron and neutron study
}

\author{
Vladislav Kochetov ${ }^{1,2} \odot$, Martin J Mühlbauer ${ }^{3,4}$, Alexander Schökel ${ }^{1,5}$ (], \\ Torben Fischer ${ }^{6}$, Timo Müller ${ }^{5}$, Michael Hofmann ${ }^{1}{ }^{1}$, Peter Staron ${ }^{6}$, Ulrich \\ Lienert $^{5}$, Winfried Petry ${ }^{6}\left[\right.$ and Anatoliy Senyshyn ${ }^{1, *}$ (i) \\ ${ }^{1}$ Forschungs-Neutronenquelle Heinz Maier-Leibnitz FRM II, Technische Universität München, \\ Lichtenbergstrasse 1, D-85748 Garching b. München, Germany \\ ${ }^{2}$ Institut für Physik, Universität Rostock, A.-Einstein-Str. 23-24, 18059 Rostock, Germany \\ 3 Deutsches Patent-und Markenamt, Zweibrückenstraße 12, D-80331 München, Germany \\ ${ }^{4}$ Institute for Applied Materials (IAM), Karlsruhe Institute of Technology (KIT), \\ Hermann-von-Helmholtz-Platz 1, D-76344 Eggenstein-Leopoldshafen, Germany \\ 5 Deutsches Elektronen Synchrotron (DESY), Notkestr. 85, D-22607 Hamburg, Germany \\ ${ }^{6}$ Helmholtz-Zentrum Geesthacht, Max-Planck-Str. 1, D-21502 Geesthacht, Germany \\ E-mail: anatoliy.senyshyn@gmail.com
}

Received 7 August 2020, revised 1 November 2020

Accepted for publication 25 November 2020

Published 21 December 2020

\begin{abstract}
Diffraction and imaging using x-rays and neutrons are widely utilized in different fields of engineering, biology, chemistry and/or materials science. The additional information gained from the diffraction signal by x-ray diffraction and computed tomography (XRD-CT) can give this method a distinct advantage in materials science applications compared to classical tomography. Its active development over the last decade revealed structural details in a non-destructive way with unprecedented sensitivity. In the current contribution an attempt to adopt the well-established XRD-CT technique for neutron diffraction computed tomography (ND-CT) is reported. A specially designed 'phantom', an object displaying adaptable contrast sufficient for both XRD-CT and ND-CT, was used for method validation. The feasibility of ND-CT is demonstrated, and it is also shown that the ND-CT technique is capable to provide a non-destructive view into the interior of the 'phantom' delivering structural information consistent with a reference XRD-CT experiment.
\end{abstract}

Keywords: neutron diffraction, diffraction computed tomography, synchrotron radiation, reconstruction

(Some figures may appear in colour only in the online journal)

\section{Introduction}

Imaging methods based on different contrast mechanisms and kinds of radiation (e.g. nuclear magnetic resonance, penetration of sound, attenuation of x-ray, neutron or electron beams) are broadly used as non-destructive probes for studying objects in different fields like engineering, biology,

* Author to whom any correspondence should be addressed.

(c) (i) Original content from this work may be used under the terms of the Creative Commons Attribution 4.0 licence. Any further distribution of this work must maintain attribution to the author(s) and the title of the work, journal citation and DOI. physics etc. Attenuation-based radiography and tomography became very advanced techniques for studies of bulk samples of different kinds, where non-destructive identification of sample components is based on the attenuation for different materials composing the sample. In the case of x-rays there is a general increase of attenuation with atomic number of the composing elements, whereas neutron scattering and absorption (being isotope-specific effects) show non-linear dependence on atomic number. Therefore, absorption-based contrast for $\mathrm{x}$-rays is not very high for light or neighboring elements in the periodic table. In order to enhance it, one may combine x-ray and neutron data sets, explore 
absorption or Bragg edges at synchrotron or neutron sources [1], use different kinds of phase contrast imaging [2-4] etc. Out of the above-mentioned approaches only Bragg edge imaging and phase contrast imaging are sufficient for distinguishing structure-based effects inside the studied samples, like composition/morphology gradients, polymorphic distribution and/or disorder. These aspects are usually studied by diffraction techniques. In order to achieve spatial resolution in combination with the obtained diffraction signal, usually a fixed gauge volume is required, defined by the beam optics either using collimators in a neutron diffraction or conical slits in a synchrotron diffraction experiment. Typical gauge volumes are in the range of $0.1-100 \mathrm{~mm}^{3}$, which limits the resolution of spatially-resolved diffraction. When non-orthogonal scattering geometry is used $\left(2 \theta \neq 90^{\circ}\right)$ the gauge volume deviates from a square/rectangular shape, which further worsens the spatial resolution due to an elongation/distortion of the effective gauge volume.

To overcome the resolution limits, researchers combined $\mathrm{x}-$ ray diffraction with computed tomography by XRD-CT [5], where similar to traditional $\mathrm{X}$-ray $\mathrm{CT}$, the sample is scanned by a pencil-beam, but the diffraction picture is collected instead of the attenuation of $\mathrm{x}$-rays in the transmitted beam as demonstrated in figure 1(a). The sample is translated along an axis, perpendicular to the beam axis, while being irradiated with a highly brilliant monochromatic x-ray beam, and the scattered $\mathrm{x}$-rays are recorded with an area detector. Thus, the whole experimental data from a single sample plane results in a 4D matrix consisting of 2D diffraction patterns obtained as a function of the translation position and the sample rotation angle. Thereby, beam size and its collimation define the basic spatial resolution. Prominent progress has been achieved during the last decades in increasing spatial and time resolution $[6,7]$ : studies applying XRD-CT for structural analysis of energy materials [8,9], biological samples [10], catalysts [11-15] and fuel cells $[15,16]$ have been reported.

The majority of XRD-CT studies are nowadays performed using new generation synchrotron sources offering coherent photon beams with sizes down to nanometers and exposure times in the range of milliseconds. But to our best knowledge no mention about neutron diffraction computed tomography (ND-CT) is present in literature. The term 'diffraction neutron imaging' is usually explored in the context of Bragg edge contrast imaging as in the recent review [17]. Another close analogy is neutron diffraction topography [18] based on works $[19,20]$ back in the 1970's and summarized in a review [21]. Unfortunately, large exposure times and weak scattering signals limit this technique from becoming a routine. Nevertheless, a series of articles proposed some modifications of imaging setups using the scattered neutron signal: for example, scattered neutron radiography, pinhole neutron diffraction [22] or a technique resembling neutron topography using microchannel plates similar to this developed by Wroblewski et al [23, 24]. Recently, a successful 3D Laue neutron diffraction has been reported on very large-grained $\alpha$-Fe and tetragonal $\mathrm{YBaCuFeO}_{5}$ [25]. None of these approaches, however, fulfill the idea of diffraction $\mathrm{CT}$, namely to reconstruct the $3 \mathrm{D}$ volume (or 2D slices) of an object from a set of diffraction patterns recorded at different positions of the object in relation to the direct beam. Therefore, a demonstration of neutron diffraction computed tomography (ND-CT) is proposed, which consists of three parts:

- preparation of a 'phantom' sample for diffraction tomography experiments;

- sample and technique validation using XRD-CT and range-of-interest XRD-CT using diffraction of highenergy photons (P07 and P21.2 beamlines at PETRA III synchrotron);

- carrying out dedicated ND-CT experiments at the instrument STRESS-SPEC (research reactor FRM II at Heinz Maier-Leibnitz Zentrum).

Both XRD-CT and ND-CT data are reconstructed in the same manner using a filtered back-projection algorithm, where XRD-CT data are presented for validation of the data reconstruction.

\section{Experimental}

\subsection{Sample preparation}

For the current study a dedicated 'phantom' sample has been produced: a 3D printed aluminium (Al) cylinder of $20 \mathrm{~mm}$ in diameter and $5 \mathrm{~cm}$ in height with a complex void layout (figures 1(a) and (b)). It was filled with different powders having known crystal structure, namely table salt-sodium chloride $(\mathrm{NaCl})$, silicon $(\mathrm{Si})$ and graphite $(\mathrm{C})$. The choice of materials was motivated by their distinct crystal structures, and correspondingly different diffraction signals ${ }^{7}$.

\subsection{Lab x-ray computed tomography (x-ray-CT)}

A high-resolution phoenix $\mathrm{v} \mid$ tome|x s system was utilized for preliminary characterization of 'phantom' sample with lab $\mathrm{x}$-ray computed tomography (figure 1(c)). The acceleration voltage and current were set to $130 \mathrm{kV}$ and $100 \mu \mathrm{A}$, accordingly with the usage of both direct and transmission tubes. A CCD detector with an active area of $1000 \times 1000$ pixels and an effective pixel size of $200 \mu \mathrm{m} \times 200 \mu \mathrm{m}$ was used for data collection. 1001 projections (x-ray radiography images) were collected during full cell rotation over $360^{\circ}$, corresponding to angular difference of $0.36^{\circ}$ between two projections. Each single projection was comprised of three datasets collected with an exposure time of $2000 \mathrm{~ms}$ and averaged. Data reconstruction was performed using phoenix datos $\mid x$ software. The analysis of the reconstructed stacks was carried out using ImageJ [26].

\subsection{X-ray diffraction tomography $(X R D-C T)$}

The XRD-CT experiment was performed at the HEMS (P07) beamline at the PETRA III synchrotron (DESY, Hamburg,

\footnotetext{
${ }^{7}$ It has to be noted that the $3 \mathrm{D}$ printed $\mathrm{Al}$ frame is characterized by a highly coarse grain microstructure potentially limiting the use of narrow beams because of the limited particle statistics in Al body.
} 

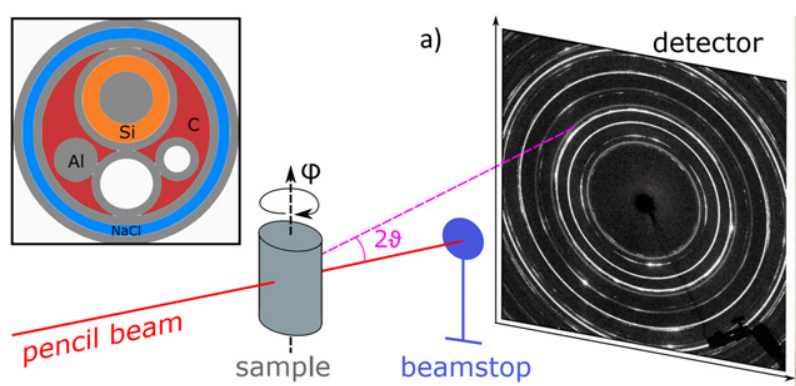
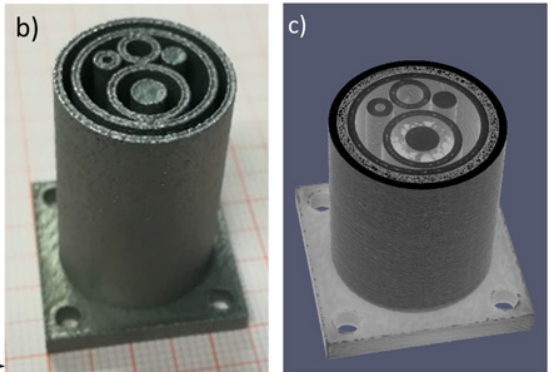

Figure 1. (a) Schematic principle of the diffraction CT, scheme of the frame partly filled with powder of different elements (red-C, orange $-\mathrm{Si}$, blue $-\mathrm{NaCl}$, grey- $\mathrm{Al}$ ), (b) 'phantom' aluminium ( $\mathrm{Al}$ ) void frame; (c) interior of 'phantom' sample as reconstructed from x-ray attenuation computed tomography.
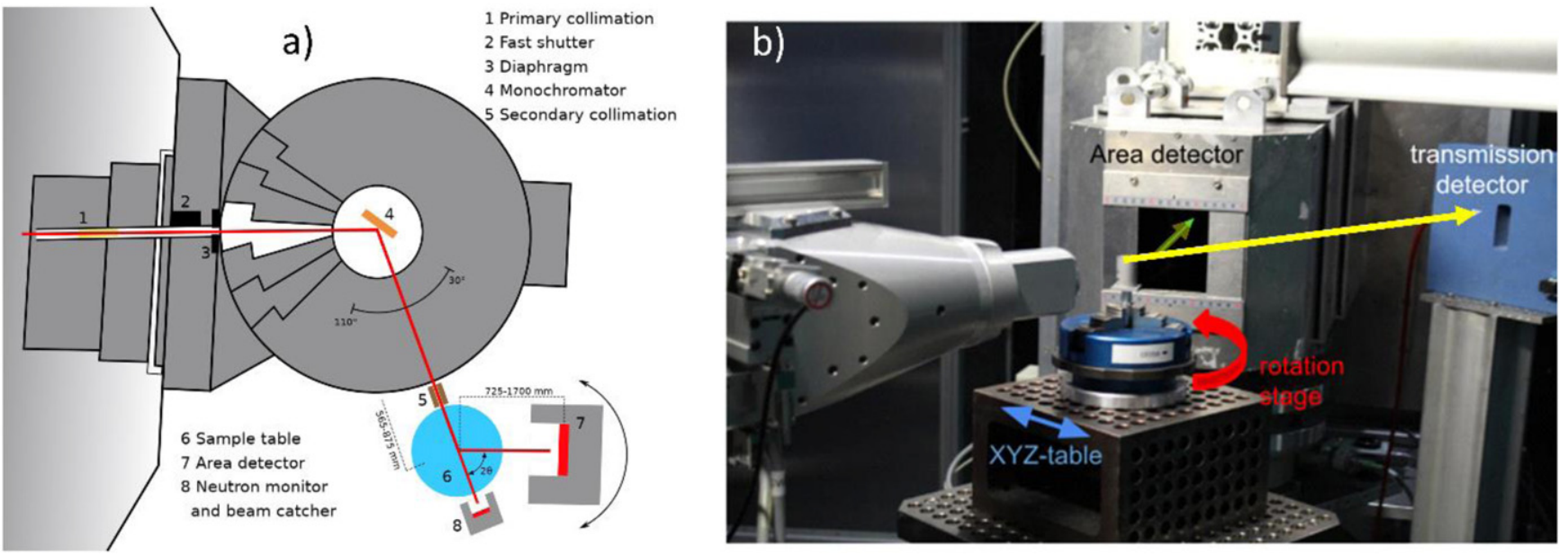

Figure 2. STRESS-SPEC: (a) schematic drawing [28], (b) experimental setup of ND-CT.

Germany) [27]. The sample was mounted on a sample goniometer enabling translation and rotation. Data were collected using a PerkinElmer XRD 1621 fast area detector with $2048 \times 2048$ pixels of $0.20 \times 0.20 \mathrm{~mm}^{2}$ size. The detector to sample distance was set to $1.217 \mathrm{~m}$. The $\mathrm{Pb}$ beamstop (installed in front of 2D detector) was equipped with a Si diode recording the intensity of the transmitted beam (as shown schematically in figure 1(a)). The beam size of $0.5 \times 0.5 \mathrm{~mm}^{2}$ was defined by a system of slits. An energy of ca. $60 \mathrm{keV}(\lambda=0.2084 \AA)$ was selected from the high-energy synchrotron beam. A NIST SRM 660a $\mathrm{LaB}_{6}$ standard powder was used as a reference for the calibration of the diffraction data.

The data collection strategy is similar to the one reported by Vamvakeros et al [12]: the object is translated in a horizontal direction perpendicular to the primary photon beam in steps of $0.2 \mathrm{~mm}$, at each translation step position, data were collected with the Perkin-Elmer detector and the Si diode in the primary beam with an exposure time of $5 \mathrm{~s}$ per data set. One translation scan consisted of 111 points in order to ensure a full coverage of the $20 \mathrm{~mm}$ wide phantom for all rotation angles, in case it would be off center. 61 of these projection lines were collected in $6^{\circ}$ steps in rotation to cover the complete range of $360^{\circ}$, resulting in a data set containing 111 translations $\times 61$ projection lines $=6771$ diffraction patterns.
An additional experiment with micrometer sized x-ray beam (ca. $5 \times 2.3 \mu \mathrm{m}$ ) was carried out at P21.2 beamline at PETRA III synchrotron. The energy of the monochromatic photon beam was $62 \mathrm{keV}$. Data were collected using a flat panel 2D detector Varex 4343CT $(2880 \times 2800$ pixels, $0.15 \times 0.15 \mathrm{~mm}^{2}$ pixel size), the distance between instrument rotation stage and detector was determined using a $\mathrm{LaB}_{6}$ reference to be $1.962 \mathrm{~m}$. Two different scans were performed:

- (FOV) scan-covering the full width of $25 \mathrm{~mm}$ (whole phantom) using $0.5 \mathrm{~mm}$ raster steps, 50 translations and 50 projections over $360^{\circ}$;

- (ROI) scan-narrow region-of-interest of $7 \mathrm{~mm}$ based on $0.05 \mathrm{~mm}$ raster, 141 translations and 144 projections over $360^{\circ}$.

In total 2500 and 20304 diffraction patterns were collected for FOV and ROI scans, accordingly.

\subsection{Neutron diffraction tomography (ND-CT)}

The neutron diffraction tomography experiment was performed at the engineering diffractometer STRESS-SPEC at research reactor FRM II (Garching b. München, Germany). The experimental setup (figure 2) for neutron diffraction CT was similar to the one with synchrotron radiation consisting of a system of slits to shape the incoming monochromatic 

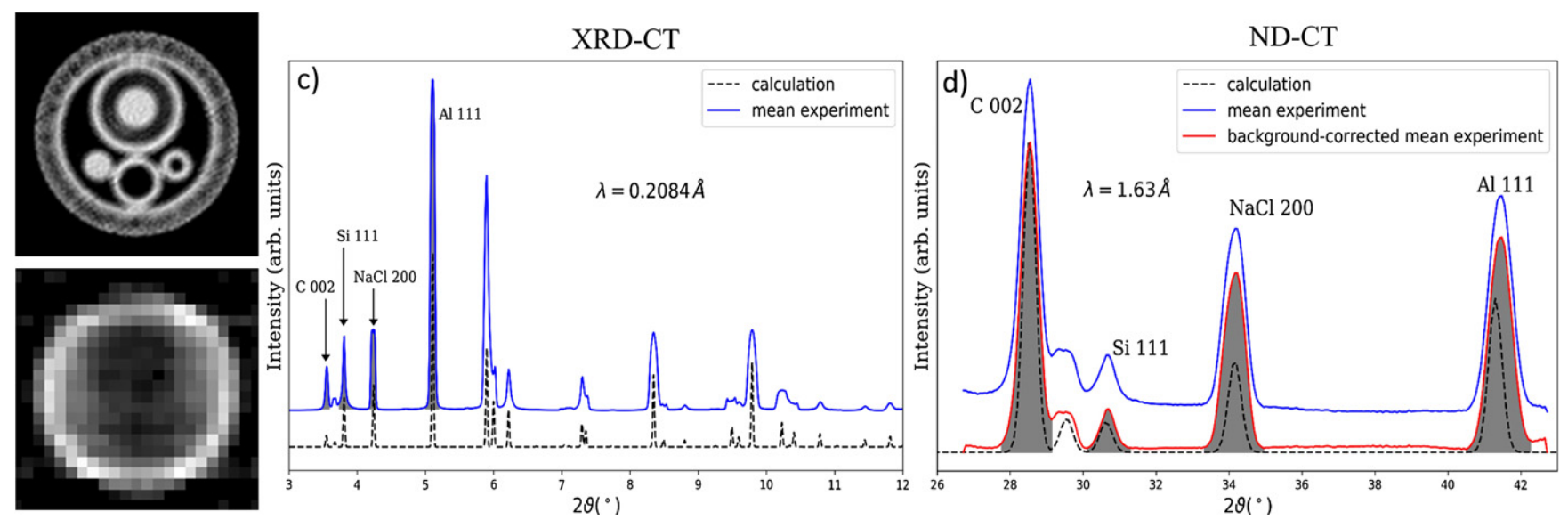

Figure 3. Results of x-ray (a) and neutron (b) absorption CT for the 'phantom' sample. Mean diffraction patterns (solid lines) of 'phantom' sample collected using synchrotron radiation (c) and neutron diffraction (d) along with theoretical patterns (dashed lines). Grey shaded regions indicate the intensities of characteristic reflections used/chosen for diffraction tomography reconstruction.

neutron beam, a sample stage to enable sample translation and rotation, a $2 \mathrm{D}{ }^{3} \mathrm{He}$ detector and a neutron monitor (uranium fission ionization chamber) mounted in front of the beamstop. A monochromatic thermal neutron beam with a wavelength of $1.63 \AA$ resulted from a $66^{\circ}$ take-off angle of a vertically focused Ge311 monochromator. In order to reach the desired spatial resolution the horizontal beam divergence was controlled by a Soller collimator of $5^{\prime}$ in front of the sample. For increased count rates the horizontal slit opening was set to $1 \mathrm{~mm}$, whilst, in accordance with the radial symmetry of the 'phantom' sample, the vertical slit opening was $10 \mathrm{~mm}$. Thus, the geometry of the setup provides the gauge volume of $10 \mathrm{~mm}^{3}$.

In analogy with the synchrotron case, the object was translated perpendicular to the neutron beam: with 25 translations having $1 \mathrm{~mm}$ step and 13 projections were taken over $360^{\circ}$ with an angular step of $30^{\circ}$. Altogether 325 diffraction patterns were collected. The $2 \mathrm{D}{ }^{3} \mathrm{He}$ multidetector was mounted at $900 \mathrm{~mm}$ distance from the sample centred at $34.5^{\circ} 2 \theta$. Its $256 \times 256 \mathrm{~mm}^{2}$ active area covered an angular range from $26.5^{\circ}$ to $42.5^{\circ} 2 \theta$. The exposure time per pattern was set to $5 \mathrm{~min}$, obtained diffraction intensities were corrected to the neutron monitor in the incident monochromatic beam.

\section{Results and discussion}

A conventional transmission tomography reconstruction of the data as obtained by Si diode and uranium fission chamber are shown in figure 3(a) for x-rays and figure 3(b) for neutrons. In the $\mathrm{X}$-ray $\mathrm{CT}$ the major contrast is caused by the bulk and dense $\mathrm{Al}$ frame, suppressing the signal from other materials inside the 'phantom'. For the neutron-based absorption CT the observed attenuation along the circumference might originate from $\mathrm{NaCl}$, since chlorine is the strongest absorber for thermal neutrons in the given 'phantom' assembly. In both x-ray and neutron cases the absorption contrast does not allow to determine the inner structure in a complete and accurate way.

Collected sets of diffraction patterns using either x-rays or neutrons were corrected for geometrical aberrations and radially integrated. In case of synchrotron data Fit2D [29] was used, whereas neutron data were reduced using SteCa [30]. Obtained mean diffraction data (averaged over the whole data set) are plotted in figures 3(c) and (d) along with theoretically generated patterns. A fair agreement between mean experimental and theoretical patterns allows us to construct sinograms for a selected Bragg reflection (interatomic spacing). Several corrections were applied accounting for possible off-centre displacements, synchrotron beam, spurious spots etc followed by a filtered back-projection algorithm implemented in Matlab [31].

Tomography reconstructions ('tomograms') for each material of the phantom obtained based on the analysis of characteristic Bragg reflections are depicted in figure 4(a) for XRDCT and figure 4(c) for ND-CT, respectively. The intensities of well-separated Bragg reflections were used as an input: (002) for $\mathrm{C}$, (111) for $\mathrm{Si},(200)$ for $\mathrm{NaCl}$ and (111) for Al. Within the given resolutions the experimental and theoretical data display reasonably good agreement.

Row (a) of figure 4 shows that full resolution XRD-CT clearly resolves the regions filled with $\mathrm{C}, \mathrm{Si}$ and $\mathrm{NaCl}$ as well as the Al housing of the 'phantom' sample. Neutron results reproduce the spatial localisation of the sample components as well. However, the results of ND-CT experiment (figure 4, row (c)) have been found to be severely resolution limited, where areas of the components' locations were significantly broadened/smeared. An attempt to mimic/resemble the resolution in synchrotron and neutron experiments was made by the reduction of XRD-CT data set dimensions to $5 \times 5$ pixels (figure 4, row (b)). Comparing rows (b) and (c) of figure 4 one can conclude a real spatial pixel resolution of ca $2.5 \times 2.5 \mathrm{~mm}^{2}$ achieved in neutron experiment against a nominal one of $1.0 \times 1.0 \mathrm{~mm}^{2}$ (figure 4 , row (c)) expected from $1 \mathrm{~mm}$ opening of horizontal sample slits. The rather coarse effective pixel size in the neutron experiment was somewhat surprising and was attributed to several factors involving counting statistics (limiting the achievable contrast in the reconstruction), non-parallel beam in neutron diffraction experiment (potentially requiring the cone correction) along with contributions from the vertical 


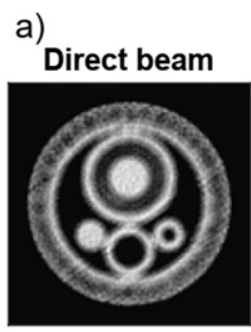

b)

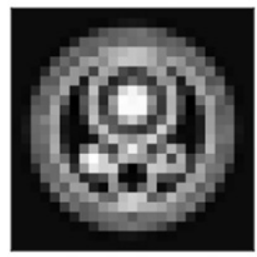

c)

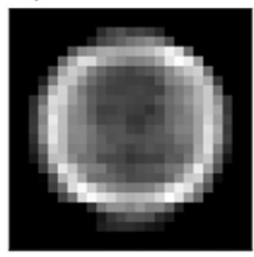

d)

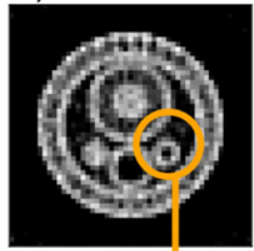

e)

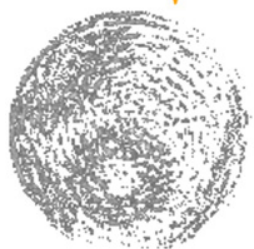

C (002)
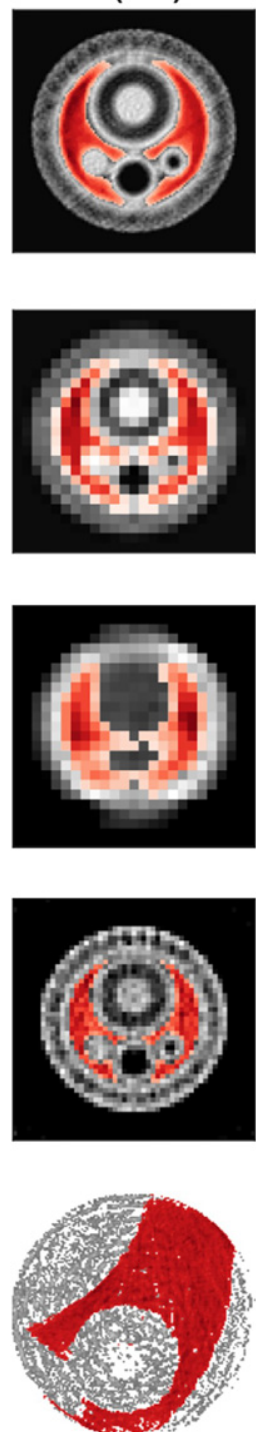

XRD-CT $0.2 \mathrm{~mm}$
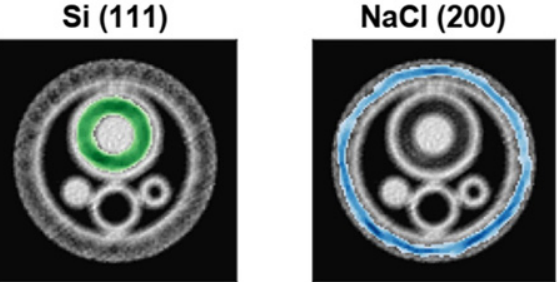

XRD-CT 0.2mm binned

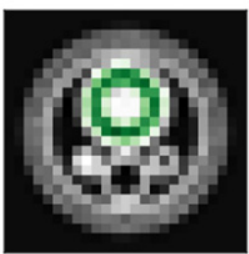

ND-CT $1 \mathrm{~mm}$

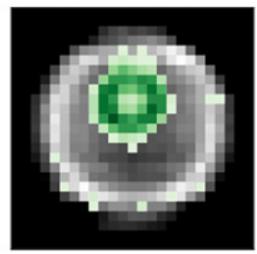

XRD-CT $0.5 \mathrm{~mm}$

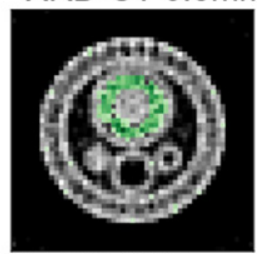

XRD-CT 0.05mm ROI


Figure 4. Tomographic reconstructions of a 'phantom' slice corresponding to XRD-CT (a), XRD-CT in a quality reduced to $25 \times 25 \mathrm{px}^{2}$ to match with ND-CT results (b), ND-CT (c), FOV scan-XRD-CT collected using $5 \mu \mathrm{m}$ beam (d), ROI scan-XRD-CT (e). All intensities were normalized to the maximal intensity in each row followed by $20 \%$ background removal.

beam component; primary beam divergence along with nonzero slit-to-sample distance result in an increased beam width at the sample.

The issue of grain size is of less importance for millimetersized beams, but becomes of increasing relevance upon attempt to further improve the resolution by the reduction of beamsize. A comparison of XRD-CT scan collected using $0.5 \mathrm{~mm}$ x-ray beam and $0.2 \mathrm{~mm}$ translation (figure 4(a)) to FOV scan using $5 \mu \mathrm{m}$ beamsize and $0.5 \mathrm{~mm}$ translation (figure 4(d)) clearly yield a less-resolved and noisy character of FOV scan. The amount of 'noise' in the reconstructed signal is even more increased in ROI scan (figure 4(e)), where one can hardly identify the contributions from different powders. This becomes especially pronounced for the signal from $\mathrm{Al}$ and $\mathrm{NaCl}$, which are characterized by the relatively coarse microstructure. Therefore a strong 'noise' observed on both attenuation and diffraction channels can be attributed to the grain contributions to the signal (either in form of attenuation and small- or wide-angle scattering of single or multiple origin).

\section{Conclusion}

A combined synchrotron/neutron diffraction tomography experiment was successfully performed on a model 'phantom' sample. Characterisation via the diffraction signal gives an improved sensitivity to crystal structure, thus opening new capabilities to distinguish different polymorphs, volumes having low/high or similar absorption coefficients, slight variations in chemical composition and/or structural modification etc. The use of neutron diffraction permits to combine the advantages of CT techniques with the unique features of thermal neutrons (isotope-sensitivity, small energy transfers, 
high penetration depth, high atomic localisation capabilities, neutron's magnetic moment) opening new perspectives for non-destructive analysis.

The synchrotron-based diffraction tomography experiment are in excellent agreement with the known layout of the phantom. The technique has a lot of potential, where the use of nanometer sized beams may help to achieve nanometer sized voxel edges. Use of highly brilliant beams and detectors with high frame rates may potentially reduce the exposure times down to microseconds, where the grain statistics will be a factor limiting spatial resolution. Dedicated strategies of data collection, reduction and reconstruction (similar to this recently reported in reference [8]) might be of great relevance for further development of XRD-CT.

The ND-CT experiment using thermal neutrons can be considered successful and in qualitative agreement with XRDCT. Although the experiment was performed at one of the most powerful engineering diffractometers in the world the neutron experiment suffered from the low count rates due to restricted gauge volume of $10 \mathrm{~mm}^{3}$ and short integration time of $5 \mathrm{~min}$. High beam collimation and neutron flux are considered to be the key ingredient for the success of ND-CT experiments requiring for further improvement of the signalto-noise ratio, spatial/resolution and experimental sensitivity. Larger area detectors along with dedicated neutron optics (e.g. Wolter optics) and extension of ND-CT technique to timeof-flight neutron diffraction may facilitate its further utilization in user experiments. With higher neutron fluxes, e.g. at the European spallation source, a boost for further development of ND-CT down to submillimeter spatial resolution can be expected. Sub millimeter-sized beams in neutron scattering are challenging, but this challenge is outweighed by the advantages of neutrons that make the technique extremely promising in terms of investigation of bulk, hydrogen-containing and light materials, isotopic contrast, distinguishing transition elements etc. These properties definitely point out the range of applications where ND-CT can be complementary to x-rays analogue or even indispensable.

\section{Acknowledgments}

The project was supported in part by FRM II and German Federal Ministry of Education and Research (BMBF project 05K16VK2 and 05K19VK3). VK thanks MAMASELF + for the granted scholarship. We acknowledge DESY (Hamburg, Germany), a member of the Helmholtz Association HGF and Heinz Maier-Leibnitz Zentrum (FRM II), for the provision of their experimental facilities. Parts of this research were carried out at PETRA III using beamline P07 and P21.2.

\section{ORCID iDs}

Vladislav Kochetov (D) https://orcid.org/0000-0002-90592948

Alexander Schökel (D) https://orcid.org/0000-0002-3680-8648 Michael Hofmann (D) https://orcid.org/0000-0003-4936-9960 Winfried Petry (D) https://orcid.org/0000-0001-5208-7070
Anatoliy Senyshyn (iD https://orcid.org/0000-0002-1473-8992

\section{References}

[1] Woracek R, Penumadu D, Kardjilov N, Hilger A, Boin M, Banhart J and Manke I 2015 Phys. Proc. $69227-36$

[2] Ludwig W, Schmidt S, Lauridsen E M and Poulsen H F 2008 J. Appl. Cryst. 41 302-9

[3] Strobl M, Treimer W, Kardjilov N, Hilger A and Zabler S 2008 Nucl. Instrum. Methods Phys. Res. B 266 181-6

[4] Zanette I, Zhou T and Burvall A et al 2014 Phys. Rev. Lett. 112 253903

[5] Harding G, Kosanetzky J and Neitzel U 1987 Med. Phys. 14 $515-25$

[6] Beale A M, Jacques S D M and Gibson E K et al 2014 Coord. Chem. Rev. 277-278 208-23

[7] Lehmann E H, Frei G, Kühne G and Boillat P 2007 Nucl. Instrum. Methods Phys. Res. A 576 389-96

[8] Finegan D P, Vamvakeros A and Tan C et al 2020 Nat. Commun. 11631

[9] Finegan D P et al 2019 Nano Lett. 19 3811-20

[10] Leemreize H, Almer J D, Stock S R and Birkedal H 2013 J. R. Soc. Interface $\mathbf{1 0} 20130319$

[11] Birkbak M E, Leemreize H, Frølich S, Stock S R and Birkedal H 2015 Nanoscale 7 18402-10

[12] Vamvakeros A, Jacques S D M, Di Michiel M, Senecal P, Middelkoop V, Cernik R J and Beale A M 2016 J. Appl. Cryst. 49 485-96

[13] Jacques S D M, Di Michiel M and Kimber S A J et al 2013 Nat. Commun. 42536

[14] Matras D, Jacques S D M, Godini H R, Khadivi M, Drnec J, Poulain A, Cernik R J and Beale A M 2018 J. Phys. Chem. C 122 2221-30

[15] Price S W T, Martin D J, Parsons A D, Sławiński W A, Vamvakeros A, Keylock S J, Beale A M and Mosselmans J F W 2017 Sci. Adv. 3 e 1602838

[16] Li T, Heenan T M M and Rabuni M F et al 2019 Nat. Commun. 101497

[17] Woracek R, Santisteban J, Fedrigo A and Strobl M 2018 Nucl. Instrum. Methods Phys. Res. A 878 141-58

[18] Tanner B K 1976 Chapter 3-contrast on X-ray topographs X-ray Diffraction Topography ed B K Tanner (Oxford: Pergamon) pp 63-99

[19] Doi K, Minakawa N, Motohashi H and Masaki N 1971 J. Appl. Cryst. 4 528-30

[20] Ando M and Hosoya S 1972 Phys. Rev. Lett. 29 281-5

[21] Schlenker M and Baruchel J 1986 Physica 137 309-19

[22] Wu W, Stoica A D and Berry K D et al 2018 Appl. Phys. Lett. 112253501

[23] Wroblewski T, Jansen E, Schäfer W and Skowronek R 1999 Nucl. Instrum. Methods Phys. Res. A 423 428-34

[24] Wroblewski T, Almanstötter J, Clauss O, Moneke M, Pirling T and Schade P 2000 Mater. Sci. Eng.: A 288 126-31

[25] Raventós M, Tovar M and Medarde M et al 2019 Sci. Rep. 9 4798

[26] Schneider C A, Rasband W S and Eliceiri K W 2012 Nat. Methods 9 671-5

[27] Schell N et al 2008 Mat. Sci. Forum 571-572 261-6

[28] Hofmann M, Schneider R, Seidl G A, Rebelo-Kornmeier J, Wimpory R C, Garbe U and Brokmeier H-G 2006 Physica B 385-386 1035-7

[29] Hammersley A P, Svensson S O, Hanfland M, Fitch A N and Hausermann D 1996 High Press. Res. 14235 -48

[30] Randau C, Garbe U and Brokmeier H-G 2011 J. Appl. Cryst. 44 641-6

[31] Tarajan E, Mühlbauer M and Senyshyn A 2018 ATZ Electron. Worldw. 13 50-5 